\title{
Current diagnosis and management strategies in pachychoroid spectrum of diseases (Review)
}

\author{
ANDREEA DANA MORARU ${ }^{1,2^{*}}$, DĂNUȚ $\operatorname{COSTIN}^{1,2}$, RADU LUCIAN MORARU $^{3 *}$, \\ MARCEL COSTULEANU $^{4 *}$ and DANIEL CONSTANTIN BRĂNIȘTEANU ${ }^{1,5}$
}

\author{
${ }^{1}$ Department of Ophthalmology, 'Grigore T. Popa' University of Medicine and Pharmacy, 700115 Iași; \\ ${ }^{2}$ Department of Ophthalmology, 'Prof. N. Oblu' Clinical Hospital, 700309 Iași; ${ }^{3}$ Department of Otorhinolaryngology, \\ 'Transmed Expert' Medical Center, 700011 Iași; ${ }^{4}$ Department of Physiopathology, 'Grigore T. Popa' University \\ of Medicine and Pharmacy, 700115 Iași; ${ }^{5}$ 'Retina Center’ Eye Clinic, 700126 Iași, Romania
}

Received June 5, 2020; Accepted July 6, 2020

DOI: $10.3892 /$ etm.2020.9094

\begin{abstract}
This review presents a systematic analysis of the literature regarding the pachychoroid disease spectrum nomenclature, diagnosis criteria and therapeutic options. Pachychoroid related diseases are a new concept, introduced into the ophthalmological nomenclature in 2013 and evolving both as concept and as classification since then. The six disorders included in this phenotype have some common characteristics (thick choroid, pachyvessels, attenuation of the choriocapillaris), but also show individual features. The classification of the pachychoroid spectrum was revised many times, with the recent addition of the focal choroidal excavation (FCE) and peripapillary pachychoroid syndrome (PPS). As the terminology is developing, so is the number of case reports and case series from the initial report in 2013 to 57 reports in 2019. This review takes into account both the current literature and the clinical experience of the authors, emphasizing the understanding of the pathogenesis and aiming to update the therapeutic options available.
\end{abstract}

\section{Contents}

1. Introduction

2. Anatomy of the choroid

3. Common characteristics of the pachychoroid disease spectrum

Correspondence to: Professor Dănuț Costin, Department of Ophthalmology, 'Grigore T. Popa' University of Medicine and Pharmacy, 16 University Street, 700115 Iași, Romania

E-mail: oftalmoconsultiasi@yahoo.ro

*Contributed equally

Key words: pachychoroid, pachyvessels, choroidal hyperpermeability, choroidal neovascular membranes, OCT angiography, anti-VEGF therapy
4. Clinical entities included in the pachychoroid disease spectrum

5. Discussion

6. Conclusions

\section{Introduction}

Pachychoroid spectrum of diseases was first taken into account in 2013 when pachychoroid pigment epitheliopathy (PPE) was described in a study by Warrow et al (1). The group of diseases analyzed in the study is characterized by retinal pigment epithelial abnormalities, accompanied by choroidal thickening.

The term 'pachy' is a Greek word ' $\pi \alpha \chi v$ ', which means thick. The pachychoroid is a relatively new concept referring to a group of ophthalmological disorders which has as major characteristics, not only a thickened choroid, but also dilated choroidal vessels and attenuation of choriochapillaris. These alterations of the choroidal structure have as a consequence the dysfunction of the retinal pigment epithelium, a layer of great importance in maintaining the normal retinal metabolism and functions and subsequent development of choroidal neovascularization (2).

The etiology of pachychoroid diseases is controversial. It is assumed that autosomal dominant heredity is implicated, together with endogenous and exogenous factors that trigger the onset of the clinical manifestations.

The pachychoroid spectrum comprises: polypoidal choroidal vasculopathy/aneurysmal type 1 neovascularization, pachychoroid neovasculopathy (PNV), PPE, focal choroidal excavation (FCE), peripapillary pachychoroid syndrome (PPS) and central serous chorioretinopathy (CSC). All these disorders have one, common pathogenic process that enables their subsequent evolution.

This review takes into account both the clinical experience of the authors and the current literature regarding the pachychoroid spectrum of diseases, emphasizing the comprehension of the pathogenic process, clinical characteristics and current therapy trends, in the context of new imaging techniques available in ophthalmology. 


\section{Anatomy of the choroid}

The choroid represents the vascular layer of the eye and it is part of the uvea together with the iris and ciliary body. It extends from the anterior ora serrata to the posterior optic nerve. The choroid provides vascular supply for the outer third of the retina. Alterations of the choroidal structure have repercussions on retinal function and can lead to the death of retinal pigment epithelial cells and the photoreceptors.

The choroid is comprised of 5 layers: Bruch Membrane, choriocapillaris, Sattler Layer - medium diameter blood vessels, Haller's Layer - large diameter blood vessels, suprachoroid lamina - a transitional zone between choroid and sclera. The choriochapillaris is important in the rapid transport of large molecules because in its structure are included large diameter capillaries and fenestrations of 700-800 nm diameter. The blood flow of the choroid is higher than that of any other tissue in the organism, 20 times higher than the retinal flow, providing oxygen and nutritive substances to the outer retina, retinal pigment epithelium, avascular fovea and the prelaminar part of the optic nerve (3).

The choroid is best studied by enhanced depth imaging-optical coherence tomography (EDI-OCT) and swept source-optical coherence tomography (SS-OCT), both techniques being able to display the deep layers of this tissue and correlate its structural and functional analysis. Optical coherence angiography (OCTA) is a new, non-invasive, 3D imaging technique, which can reconstruct the blood flow in all the vascular layers of the choroid without using contrast substances.

As viewed on OCT, choroid thickness is the greatest in the subfoveal region and it is thinner in the nasal and temporal areas. Choroidal thickness varies with age, gender, ethnical group, refractive error and axial length, but the mean thickness is considered to be between 260-300 microns (4). Some variations in the thickness of choroid are possible: it decreases with age and with a longer axial length of the eye and it temporary increases in the acute stages of severe posterior uveitis (multifocal choroiditis, multiple white dot syndrome, Vogt-Koyanagi-Harada syndrome) $(5,6)$.

\section{Common characteristics of the pachychoroid disease spectrum}

Advances in OCT imaging technology allowed a better understanding of the pathological processes implicated in the manifestation of various ophthalmological diseases. Using EDI-OCT or SS-OCT it is possible to quantify the choroidal thickness. Thus, a new group of diseases was described, named generally 'pachychoroid diseases' which have a sustained, focal or diffuse increase in choroidal thickness over 300 microns, as common characteristic. The thick choroid is due to the dilatation of Haller's layer vessels, accompanied by subsequent hyperpermeability. Both the vessels in Satler's layer and the choriocapilaris have reduced thickness.

In mostcases the subfoveal choroidal thickness is normal, but it is increased in the extrafoveal area by more than 50 microns. Choroidal thickness, although frequently encountered on OCT, is not the major diagnostic criteria. Each of the diseases included in the pachychoroid spectrum has specific morphological alteration important for establishing the diagnostic. Furthermore, according to Dansingani et al (7), cases that show increased choroidal thickness on OCT, without any changes in the retina or pigmentary epithelium are considered as uncomplicated pachychoroid.

The dilated vessels in the Haller layer were named pachyvessels and can be observed on OCT images as large and hyporeflective lumens (Fig. 1). Such vessels were reported to be present, by Yang et al (8), in central serous corioretinopathy, pachychoroid vasculopathy, PPS and FCE. These vessels do not reduce their calipers towards the macula, but terminate abruptly. The layout of pachy vessels may be diffuse or focal and they are situated in the areas of maximum choroidal thickness and choriocapilaris thinning, and are accompanied by retinal morphofunctional changes.

An important feature for pachychoroid disease spectrum is the attenuation of the choriocapillaris and the intermediate caliper vessels in the Satler layer. Due to the thinning of these two vascular layers, some eyes may have a normal choroidal thickness. This attenuation of small and intermediate diameter vessels may signify choroidal ischemia and it is associated, in some cases with retinal outer nuclear layer atrophy, as seen on OCT images and possibly with photoreceptors degeneration.

In order to assess the role of choroidal vasculature features in the development of the disease, automated software that segments the luminal choroidal area from the stromal area on OCT images, was developed by Agarwal et al (9). An increased vascular area was associated with pachychoroid disorders. In a recent study, Saito et al (10) demonstrated that choroidal hyperperfusion has an important role in the development of central serous chorioretinopathy (CSC) and pachychoroid pigment epitheliopathy (PPE), by measuring the choroidal blood flow velocity.

\section{Clinical entities included in the pachychoroid disease spectrum}

Pachychoroid pigment epitheliopathy. PPE is considered to be a precursor of CSC. In most cases the patients are asymptomatic, presenting changes in the structure of the retinal pigment epithelium, but without the clinical manifestations specific to CSC.

Some patients diagnosed with unilateral CSC have alterations specific to PPE in the contralateral eye, which might be proof that PPE represents the first stage of CSC. Some studies report that PPE may evolve to another entity from the pachychoroid spectrum as choroidal neovascular membranes appear, with or without anevrismal lesions (11).

On spectral OCT these patients do not present neurosensory retinal detachment, but it is possible to encounter small pigment epithelium detachments, mottling of the retinal pigment epithelium in all overlying areas of increased choroidal thickness (Fig. 1). Often, the PPE is confused with the onset of age-related macular degeneration (AMD), but soft drusens are absent in these cases and the OCT anomalies are present at a younger age than those diagnosed in AMD.

OCT angiography is useful in documenting the early changes as it is possible to analyze the increased choroidal thickness, the presence of pachyveins in Haller's layer and the thinning in Sattler's layer and choriocapillaris. A recent 

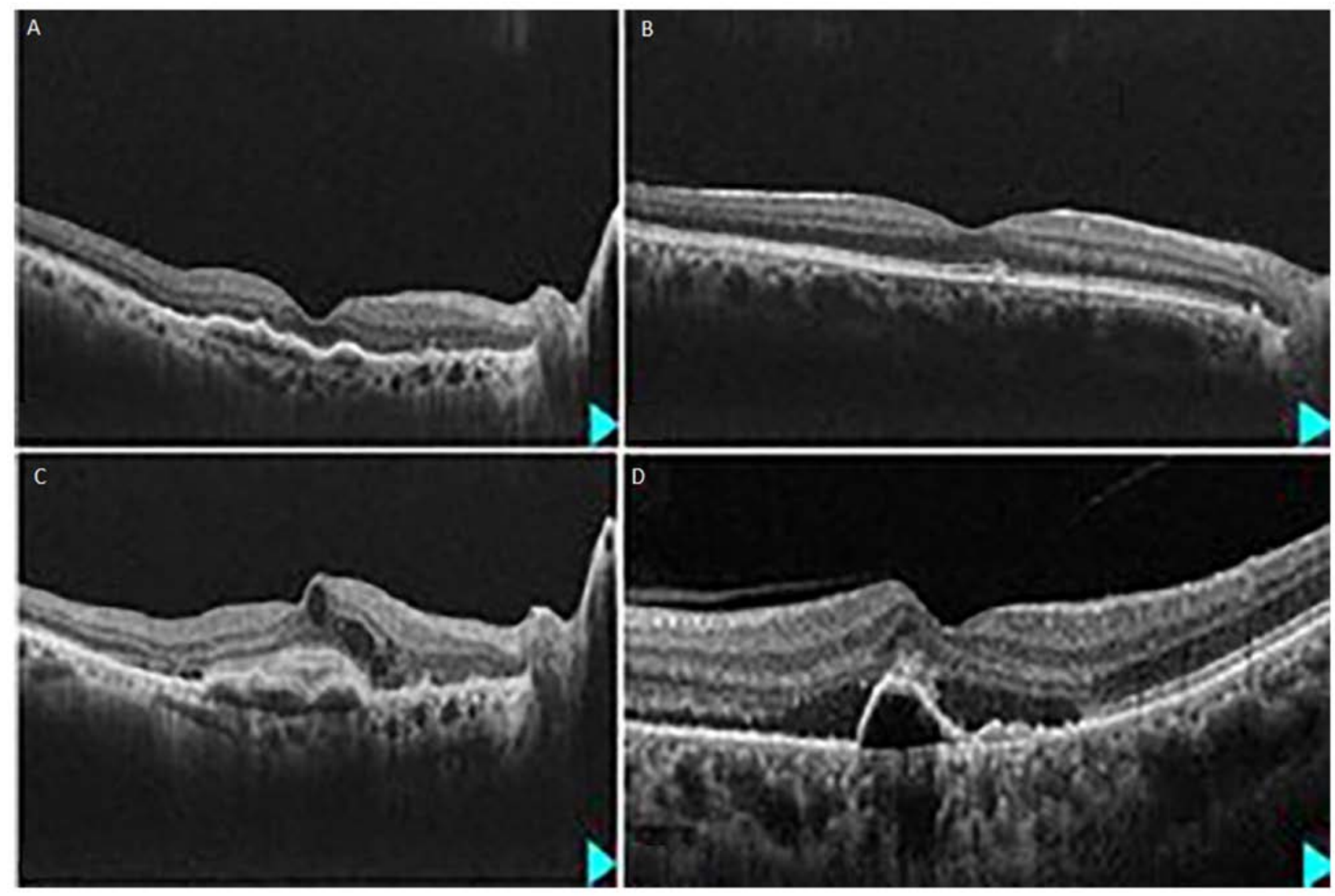

Figure 1. Pachychoroid disease spectrum characteristics: (A) OCT image of pachyvessels - thickened choroid and dilated choroidal vessels; (B) OCT image of PPE - thickened choroid and RPE alterations; (C) OCT image of PNV - irregular RPE detachment over thickened choroid and 'double layer sign' - visibility of both RPE and Bruch's membrane; (D) OCT image of PCV- thickened choroid, subretinal fluid and narrow, irregular RPE detachment with polypoid aspect. OCT, optical coherence tomography; PPE, pachychoroid pigment epitheliopathy; PNV, pachychoroid neovasculopathy.

study by Sakurada et al demonstrated that there is a direct relationship between choroidal hyperpermeability, reduced choriocapilaris flow density and increased choroidal thickness in PPE patients (12).

Central serous chorioretinopathy. CSC is a disorder that is commonly affecting young or middle-aged males that present one or more of the following risk factors: stress, autoimmune diseases, hypertension, alcohol or tobacco consumers, gastroesophageal reflux disease, users of corticosteroids, antacids, antihistamines or amphetamines. The characteristic symptoms are blurred vision, metamorphopsias, central scotoma, hyperopic shift. Most cases are auto-limited and resolve within 6 months, but the disease may be recurrent (13).

OCT examination reveals hyporeflective subretinal fluid, one or more retinal pigment epithelial detachments within or outside the subretinal fluid area, thick choroid. The exudation under the retina is the result of the abnormalities that affect the RPE and Bruch's membrane and allow an increased permeability at the level of the choroid. A pigment epithelium detachment (PED) is visible on OCT in many cases. Hwang et al (14) showed that a flat, irregular PED that accompanies CSC is a risk factor for longer evolution of the symptoms and the development of a subfoveal neovascular membrane, thus including these features in the pachychoroid neovasculopathy (PNV) phenotype.
In some cases the disease becomes chronic and typical OCT findings in these cases includes persistent subretinal fluid, RPE degeneration, cystoid edema, elongation of outer segments of photoreceptors. As the disorder progresses, atrophy affects the outer retina.

OCT angiography has replaced fluorescein angiography in the assessment of CSC. Fluorescein angiography shows leakage, corresponding to the RPE detachments and is classically described as 'smokestack' pattern. OCT angiography can reveal the areas of choroid dysfunction, hypoperfusion of choriocapillaris and the hyperperfusion present in the surrounding area.

CSC is considered the second stage of the pachychoroid disease spectrum. Some studies revealed that the choroid changes are the main starting point for the physiopathological process. Due to recent advances in technology, it is possible to observe the dilated vessels in the choroid and compare the pathological findings with the asymptomatic fellow eye $(8,15)$. A new analysis by Demirel et al (16) of the choroidal features specific to pachychoroid spectrum diseases revealed that the choriocapillaris vessel density is much lower in CSC compared with uncomplicated pachychoroid and PPE.

Pachychoroid neovasculopathy. PNV may be considered a late complication of the pigment epitheliopathy and CSC. This entity of the pachychoroid spectrum is characterized by the 
development of type 1 neovascular membrane, located under the RPE and overlying areas of thick choroid and dilated choroidal vessels.

The correspondence between the neovascular membrane location and the abnormalities inside the choroid is important for differentiating the neovasculopathy from the neovascular form of AMD. Usually, the AMD patients are older and have been diagnosed before with drusens, but the patients with PNV may have presented previously with a macular aspect consistent with PPE. Sometimes these patients are diagnosed with a neovascular membrane of unknown cause. In a recent comparative study, Arf et al (17) concluded that the neovascular membranes visualized by OCT angiography in PNV patients are similar to those of type I AMD patients, but appear in younger patients and are associated with thicker choroids.

The differential diagnosis between these abnormalities is difficult both by clinical examination and with the aid of fluorescein angiography, which shows leakage in either of the situations. OCT and OCT angiography may help in establishing the correct diagnosis, as the morphology presented by these imaging techniques are pathognomonic. The hyperpermeability exerted by the thick choroid determines choriochapilaris and Sattler's layer ischemia which results in proliferation and ingrowth of the Haller's layer vessels. In 2019, Matsumo et al (18) demonstrated that choroidal dilated veins are a characteristic of PNV and this feature has as a consequence an alteration of the venous drainage routes due to the appearance of new anastomosis between the superior and inferior vortex veins.

On OCT it is possible to observe an irregular detachment of the RPE, with an almost flat or shallow profile and an inhomogeneous aspect beneath it, which is relevant for the neovascularization located above an area of pachychoroid (Fig. 1). A 'double layer sign' may be present, representing the visibility of both RPE and Bruch's membrane. OCT angiography shows a network of vessels, typical for an occult neovascular membrane, between the RPE and Bruch membrane (19).

The association between the irregular PED, CSC and the further development of PNV seems to have a genetic determinism. Hosoda et al (20) recently identified two susceptibility loci for pachychoroid related CSC, both expressed mostly in the choroidal tissue. One of these genes is also related to the development of AMD, showing that a common pathway is possible for both diseases.

Polypoidal choroidal vasculopathy. Polypoidal choroidal vasculopathy, also known as aneurysmal type I neovascularization, was described in a study by Imamura et al (21) as an area of choroidal capillaries proliferation under the RPE, which develops aneurysms, similar to polyps, at its tip. The proliferation than evolves to serous or hemorrhagic detachment of the RPE.

This form of the disease, together with the PNV, represents the most advanced stages of the pachychoroid disease spectrum. It is considered that 1 in 10 patients with neovascular AMD is falsely diagnosed and it actually represents a pachychoroid patient (22).

A recent analysis of the characteristics of the disease revealed that the aneurysms and the neovascular network are located between the inner Bruch membrane and the RPE and have the potential to bleed in this tight space, thus the 'polyp' designation is not accurate (23).

The development of the aneurysmal dilations is the starting point for the exudative process, which evolves in a cyclical manner. The exudation is auto-limited due to the thrombosis of the aneurysm, but the vascular network continues to grow for many years, generating new dilations at the edge of the original lesion and thus the exudative process is recurrent and leads to chronic changes. The relapsing manner in which the pathological process evolves is a stimulus for the growth of the neovascular membrane, contributing to an additional exudation (24).

As opposed to the AMD patients, in the polypoidal choroidal vasculopathy cases, EDI-OCT and SS-OCT show a thickened choroid, choroidal hyperpermeability, subretinal fluid and narrow, irregular RPE detachments with polypoid aspect (Fig. 1). A large study on polypoidal choroidal vasculopathy eyes, conducted by Lee et al (25), demonstrated that although the choroidal thickness may have large variability in these cases, the majority of the patients have pachyvessels as feeding sources of the aneurysms and attenuation of the inner choroid. A new comparative study revealed that the fellow eyes of the PCV affected ones had a Haller layer to subfoveal choroidal thickness ratio comprised between the ratio of the abnormal and the normal eyes. Furthermore, during an observation period of 5 years, the choroidal vascularity index, defined as luminal to total choroidal area ratio, changed in these eyes and $16 \%$ developed PCV specific lesions (26).

OCT angiography is able to show the network of filamentous neovessels occupying the outer retina to choriocapillaris layer (ORCC). A retrospective case series, by Fujita et al (27), which included OCT angiography data from 54 eyes, confirmed that OCTA can replace indocyanine green angiography in detecting the polypoid lesions.

Peripapillary pachychoroid syndrome. This syndrome was recently described as an entity of the pachychoroid disease spectrum in which the thickened choroid is located in the proximity of the optic disc (28).

As a consequence, the subretinal and intraretinal fluid is distributed in the nasal macular and peripapillary area. Some cases may associate pigment epitheliopathy, pigment epithelial detachment or optic disc edema. Most of the eyes have hyperopic refraction and short axial lengths. Caution is needed in order to establish a correct differential diagnosis between the PPS and a series of inflammatory diseases such as posterior uveitis or neuro-ophthalmologic disorders.

Although described as a sporadic association, Govetto et al (29) published a case study in which the PPS was related to pulmonary arterial hypertension. In this case, choroidal ischemia is thought to have been caused by hemodynamic disturbances due to increased central venous pressure (30).

Focal choroidal excavation. The FCE may be encountered as a single manifestation of the pachychoroid disease in an eye, or it may be associated with one of the other entities included in this phenotype. There are reports of the association of the FCE with CSC, PNV and polypoidal choroidal vasculopathy, either in the presenting eye or in the contralateral eye $(31,32)$. 
Most of these patients are asymptomatic or only have minor visual symptoms, blurring and metamorphopsias and present myopic refraction. The disease manifests in patients younger than those diagnosed with AMD and without a history of scleral staphyloma.

On OCT it is possible to observe areas of choroidal excavation which can manifest under two patterns: with or without contact between the photoreceptors and the retinal pigment epithelium. Subretinal fluid may be present at this level. The choroidal excavation is associated with pachychoroid features. A study by Rajabian et al (33) analyzed the OCT angiography features of 28 eyes with FCE and concluded that choroidal stroma was reduced in areas close to FCE and both the choriocapillaris and the deep plexus had a lower vessel density compared with normal eyes. The authors suggested that the occurrence of FCE is possible due to the weakening of the choroidal architecture.

Another possible pathogenic theory may be that the abnormalities represent areas of scarring of the connective tissue in the choroid, probably of inflammatory nature, that leads to compression on the choriocapillaris, subsequent ischemia and predisposition to neovascular membrane development (31).

Banaee et al (34) described an FCE case that developed in the vicinity of a pachyvessel, in an eye with pachychoroid characteristics, leading to the disappearance of the vessel and proposed as a pathogenic mechanism the thrombosis of the pachyvessel.

\section{Discussion}

As most studies show, the main pathogenic mechanism leading to all the abnormalities associated with pachychoroid disease manifestation is the choroidal dysfunction. The increased choriocapillaris permeability generates, in time, the attenuation of this structure with pigmentary changes in the RPE and VEGF expression. As a result, the disease progresses to neovascular membranes and exudation (23). The occurrence of the neovascular membranes and their evolution is different from those associated with AMD, as choriocapillaris chronic inflammation and attenuation are the triggers of the disease (35).

The pathogenesis of the pachychoroid disease remains controversial, but there are some suppositions that an altered steroid metabolism is involved. It is known that the choroid expresses receptors for mineralocorticoid and the continuous stimulation of these receptors leads to an increase in its thickness, as Zhao et al (36) demonstrated in their study. Ersoz et al (37) reported that prolonged administration of corticosteroids may have as a result occurrence of PPE features, followed by CSC development, confirming the supposition that dexamethasone intravitreal implants alter choroidal functionality.

Another theory states that the increased hydrostatic pressure at the level of the Bruch membrane, RPE complex is the main factor to blame in the development of the pigment epithelium and neurosensory retina detachments. As a result of the choriocapillaris attenuation and the subsequent ischemia, there is an overexpression of pro-angiogenic factors that stimulates the development of a choroidal neovascular membrane. At the same time, the increase in volume of the
Haller's layer vessels and the mechanical pressure on the RPE causes atrophy, pigmentary changes and ruptures of the Bruch membrane, facilitating the occurrence of CNV.

There are some suppositions about the genetic phenotype that may be common to all the entities included in the pachychoroid spectrum. Also, some risk genes have been found to be common with those of AMD, but although these genes have a role in the occurrence of the neovascular membranes, they do not influence the pachychoroid features (38). In some patients presenting the characteristic genetic features of the pachychoroid, the RPE can eliminate the excess fluid and the disease is not manifest. These cases are considered to be an uncomplicated pachychoroid phenotype. In the rest of the cases, the function of the RPE is altered and the disease manifests as either minor changes at this level (PPE) or evolves to exudation (CSC), loss of the vascular layer (choriocapillaris), ischemia and neovascularization (PCV, PNV). Thus the different entities of the pachychoroid spectrum appear to be stages of the same disease, but the evolution from one stage to another is not mandatory.

The therapeutic arsenal for pachychoroid disease does not include any novelties. Asymptomatic cases can be observed. In this category are included most CSC, FCE and PPE cases.

The subretinal fluid is spontaneously resorbed in the majority of acute CSC cases. Early treatment is necessary if the visual acuity is very low, or the affected eye is the only functional eye. Subthreshold micropulse laser photocoagulation is one of the current options of treatment, able to seal the RPE defect and thus prevent the accumulation of subretinal fluid.

Photodynamic therapy using verteporfirin (PDT) may be used for chronic CSC, but some adverse effects have been reported, such as CNV formation and alterations of the electroretinography aspect. Some studies suggest that half-dose PDT is safer, but it is effective in reducing choroidal thickness, especially at the level of Haller layer (39).

The use of anti-VEGF agents is controversial in CSC. It is postulated that choroidal ischemia accompanies CSC, with a subsequent rise in the level of VEGF, thus the administration of anti-VEGF agents is beneficial. Chhablani et al (40) showed that in most cases of FCE, PNV and polypoid choroidal vasculopathy, the administration of intravitreal anti-VEGF agents resulted in improved visual acuity and resolution of subretinal and intraretinal fluid. However, the effect of anti-VEGF agents on persistent subretinal fluid and macular edema secondary to CSC was not clearly demonstrated in two large trials $(41,42)$. Authors such as Schworm et al (43) and Sacconi et al (44) concluded that intravitreal anti-VEGF therapy is useful both in fluid resorption and visual acuity gain, but the vessel density in the neovascular membrane does not change after treatment, thus supporting the theory that arteriogenesis is the main pathogenic mechanism in pachychoroid related CNV.

The activity of PNV is not only modulated by the development of the neovascular membrane and the implicit rise in VEGF levels, but also by the choroidal structural alterations. This is the reason for a weaker response and a modest response to intravitreal therapy compared with non-pachychoroid related CNV (45). PDT is safe and has a better visual outcome when used in PNV eyes refractory to anti-VEGF. Lee and Lee (46) showed that adjunctive photodynamic therapy in 
eyes non-responsive to anti-VEGF monotherapy resulted in an almost complete subretinal fluid resorption and a better visual outcome (46).

As PCV tends to have a high risk of severe visual acuity loss due to either persistent serous detachment of the macula that leads to RPE and sensory retina atrophy or acute rupture and hemorrhage of the polyp, it is justified to commence the anti-VEGF therapy early in the evolution of the disease. The EVEREST study showed that, although PDT is more efficient in inducing regression of the neovascular membrane than anti-VEGF therapy, more visual acuity was gained after the latter (47). Nevertheless, PCV eyes with pachychoroid have a weaker response to intravitreal therapy than those with normal choroidal thickness (48). Baek et al (49) considered that an explanation might be the fact that the VEGF level of PCV eyes with pachychoroid is lower than that of normal choroid eyes.

In terms of the most efficient anti-VEGF agent, there does not seem to be much difference between bevacizumab, ranibizumab and aflibercept regarding the improvement in visual acuity, the number of injections and diminishing of the retinal thickness. Some studies reported that aflibercept has a higher closure rate of the polyp and is efficient in those cases that do not respond to ranibizumab (50,51). Koizumi et al (52) showed that aflibercept can penetrate the choroid and to reduce its thickness. Aflibercept has a vasoconstrictor effect on choroidal vessels and reduces choroidal hyperpermeability, but may lead to outer retinal atrophy (53).

Combined therapy (PDT and anti-VEGF agents) is more efficient in controlling the neovascular membranes than monotherapy. This effect is due to both the diminishing of exudation by the intravitreal anti-VEGF agent and the thrombosis induced by PDT in the neovessels (54-58).

Almost one-third of the PCV eyes suffer from submacular hemorrhage during the first 10 years after diagnosis, rendering an unfavorable visual outcome in these cases. The combination of pneumatic displacement of the hemorrhage associated with intravitreal anti-VEGF seems to be the best therapy for improving the functional outcome (59).

\section{Conclusions}

Since the inclusion of the pachychoroid in the ophthalmologic nomenclature in 2013, significant progress was made in understanding the pathogenesis of the diseases in this group, improving the diagnosis tools available to the clinician and expanding the currently accepted classification. Additional research is needed in order to better understand the mechanisms that determine the development of a certain manifestation of the disease and the progression from one form to another, to improve the therapeutic management and target the pathogenic mechanism of each clinical form.

\section{Acknowledgements}

Not applicable.

\section{Funding}

No funding was received.

\section{Availability of data and materials}

Not applicable.

\section{Authors' contributions}

ADM and DCB contributed to the design of the study, participated in the entire review process and prepared the manuscript. DC and RLM contributed to the literature research and the analysis and critical interpretation of the data. ADM, DCB and $\mathrm{MC}$ conceived the review and revised the manuscript. DC gave the final approval for the publication of the manuscript. All authors read and approved the final version of the manuscript.

\section{Ethics approval and consent to participate}

Not applicable.

\section{Patient consent for publication}

Not applicable.

\section{Competing interests}

All the authors declare that they have no competing interests.

\section{References}

1. Warrow DJ, Hoang QV and Freund KB: Pachychoroid pigment epitheliopathy. Retina 33: 1659-1672, 2013.

2. Maranduca MA, Branisteanu D, Serban DN, Branisteanu DC, Stoleriu G, Manolache N and Serban IL: Synthesis and physiological implications of melanic pigments. Oncol Lett 17: 4183-4187, 2019.

3. Mrejen S and Spaide RF: Optical coherence tomography: Imaging of the choroid and beyond. Surv Ophthalmol 58: 387-429, 2013.

4. Lehmann M, Bousquet E, Beydoun T and Behar-Cohen F: Pachychoroid: An inherited condition? Retina 35: 10-16, 2015.

5. Goldenberg D, Moisseiev E, Goldstein M, Loewenstein A and Barak A: Enhanced depth imaging optical coherence tomography: Choroidal thickness and correlations with age, refractive error, and axial length. Ophthalmic Surg Lasers Imaging 43: 296-301, 2012.

6. Margolis R and Spaide RF: A pilot study of enhanced depth imaging optical coherence tomography of the choroid in normal eyes. Am J Ophthalmol 147: 811-815, 2009.

7. Dansingani KK, Balaratnasingam $\mathrm{C}$, Naysan $\mathrm{J}$ and Freund KB: En face imaging of pachychoroid spectrum disorders with sweptsource optical coherence tomography. Retina 36: 499-516, 2016.

8. Yang L, Jonas JB and Wei W: Choroidal vessel diameter in central serous chorioretinopathy. Acta Ophthalmol 91: e358-e362, 2013.

9. Agrawal R, Gupta P, Tan KA, Cheung CM, Wong TY and Cheng CY: Choroidal vascularity index as a measure of vascular status of the choroid: Measurements in healthy eyes from a population-based study. Sci Rep 6: 21090, 2016.

10. Saito W, Hashimoto Y, Hirooka K and Ishida S: Changes in choroidal blood flow velocity in patients diagnosed with central serous chorioretinopathy during follow-up for pachychoroid pigment epitheliopathy. Am J Ophthalmol Case Rep 18: 100651, 2020.

11. Pang CE and Freund KB: Pachychoroid neovasculopathy. Retina 35: 1-9, 2015.

12. Sakurada Y, Fragiotta S, Leong BCS, Parikh R, Hussnain SA and Freund KB: Relationship between choroidal vascular hyperpermeability, choriocapillaris flow density and choroidal thickness in eyes with pachychoroid pigment epitheliopathy. Retina 40: 657-662, 2020.

13. Brănişteanu DE, Pintilie A, Andreş LE, Dimitriu A, Oanţă A, Stoleriu G and Brănişteanu DC: Ethiopathogenic hypotheses in lichen planus. Rev Med Chir Soc Med Nat Iasi 120: 760-767, 2016. 
14. Hwang H, Kim JY, Kim KT, Chae JB and Kim DY: Flat irregular pigment epithelium detachment in central serous chorioretinopathy: A form of pachychoroid neovasculopathy? Retina Oct 1, 2019 (Epub ahead of print). doi: 10.1097/ IAE.0000000000002662.

15. Imamura Y, Fujiwara T, Margolis R and Spaide RF: Enhanced depth imaging optical coherence tomography of the choroid in central serous chorioretinopathy. Retina 29: 1469-1473, 2009.

16. Demirel S, Değirmenci MFK, Batığlu F and Özmert E: Evaluation of the choroidal features in pachychoroid spectrum diseases by optical coherence tomography and optical coherence tomography angiography. Eur J Ophthalmol 4: 1120672119887095, 2019.

17. Arf S, Sayman Muslubas I, Hocaoglu M, Ersoz MG and Karacorlu M: Features of neovascularization in pachychoroid neovasculopathy compared with type 1 neovascular age-related macular degeneration on optical coherence tomography angiography. Jpn J Ophthalmol 64: 257-264, 2020.

18. Matsumoto H, Kishi S, Mukai R and Akiyama H: Remodeling of macular vortex veins in pachychoroid neovasculopathy. Sci Rep 9: 14689, 2019.

19. Bousquet E, Bonnin S, Mrejen S, Krivosic V, Tadayoni R and Gaudric A: Tadayoni R and Gaudric A: Optical coherence tomography angiography of flat irregular pigment epithelium detachment in chronic central serous chorioretinopathy. Retina 38: 629-638, 2018

20. Hosoda Y, Miyake M, Schellevis RL, Boon CJF, Hoyng CB Miki A, Meguro A, Sakurada Y, Yoneyama S, Takasago Y, et al: Genome-wide association analyses identify two susceptibility loci for pachychoroid disease central serous chorioretinopathy. Commun Biol 2: 468, 2019.

21. Imamura Y, Engelbert M, Iida T, Freund KB and Yannuzzi LA: Polypoidal choroidal vasculopathy: A review. Surv Ophthalmol 55: 501-515, 2010.

22. Freund KB, Zweifel SA and Engelbert M: Do we need a new classification for choroidal neovascularization in age-related macular degeneration? Retina 30: 1333-1349, 2010.

23. Dansingani KK, Gal-Or O, Sadda SR, Yannuzzi LA and Freund KB Understanding aneurysmal type 1 neovascularization (polypoidal choroidal vasculopathy): A lesson in the taxonomy of 'expanded spectra' - a review. Clin Exp Ophthalmol 46: 189-200, 2018.

24. Uyama M, Wada M, Nagai Y, Matsubara T, Matsunaga H, Fukushima I, Takahashi K and Matsumura M: Polypoidal choroidal vasculopathy: Natural history. Am J Ophthalmol 133: 639-648, 2002.

25. Lee WK, Baek J, Dansingani KK, Lee JH and Freund KB Choroidal morphology in eyes with polypoidal choroidal vasculopathy and normal or subnormal subfoveal choroidal thickness. Retina 36 (Suppl 1): S73-S82, 2016.

26. Lee K, Park JH, Park YG and Park YH: Analysis of choroidal thickness and vascularity in patients with unilateral polypoidal choroidal vasculopathy. Graefes Arch Clin Exp Ophthalmol 258: $1157-1164,2020$.

27. Fujita A,Kataoka K, Takeuchi J,Nakano Y,Horiguchi E, Kaneko H, Ito $\mathrm{Y}$ and Terasaki H: Diagnostic characteristics of polypoidal choroidal vasculopathy based on B-scan swept-source optical coherence tomography angiography and its interrater agreement compared with indocyanine green angiography. Retina: Jan 20 , 2020 (Epub ahead of print). doi: 10.1097/IAE.0000000000002760.

28. Phasukkijwatana N, Freund KB, Dolz-Marco R, Al-Sheikh M, Keane PA, Egan CA, Randhawa S, Stewart JM, Liu Q, Hunyor AP, et al: Peripapillary pachychoroid syndrome. Retina 38: 1652-1667, 2018.

29. Govetto A, Sarraf D and Scialdone A: 'Hide and seek' neurosensory retinal detachments in peripapillary pachychoroid syndrome associated with pulmonary arterial hypertension. Retin Cases Brief Rep: Nov 21, 2019 (Epub ahead of print). doi: 10.1097/ICB.0000000000000942.

30. Branisteanu DE, Nichifor M, Dorobat CM, Brănisteanu DC, Petrariu FD, Molodoi AD, Radu DC and Boda D: Use of textile biomaterials for the topic treatment of chronic venous disease. Rom Biotechnol Lett 20: 10618-10625, 2015.

31. Ellabban AA, Tsujikawa A, Ooto S, Yamashiro K, Oishi A, Nakata I, Miyake M, Akagi-Kurashige Y, Ueda-Arakawa N, Arichika S, et al: Focal choroidal excavation in eyes with central serous chorioretinopathy. Am J Ophthalmol 156: 673-683, 2013.

32. Lim FP, Wong CW, Loh BK, Chan CM, Yeo I, Lee SY, Mathur R, Wong D, Wong TY and Cheung CM: Prevalence and clinical correlates of focal choroidal excavation in eyes with age-related macular degeneration, polypoidal choroidal vasculopathy and central serous chorioretinopathy. Br J Ophthalmol 100 918-923, 2016.
33. Rajabian F, Arrigo A, Jampol LM, Mercuri S, Introini U, Bandello F and Battaglia Parodi M: Optical coherence tomography angiography features of focal choroidal excavation and the choroidal stroma variations with occurrence of excavation. Retina: 2020 Jan 23, 2020 (Epub ahead of print). doi: 10.1097/IAE.0000000000002765.

34. Banaee T, Lyons LJ and El-Annan J: Development of focal choroidal excavation in non-neovascular age related macular degeneration with pachy-choroid features. J Curr Ophthalmol 31: 454-457, 2019.

35. Kitaya N, Nagaoka T, Hikichi T, Sugawara R, Fukui K, Ishiko S and Yoshida A: Features of abnormal choroidal circulation in central serous chorioretinopathy. Br J Ophthalmol 87: 709-712, 2003.

36. Zhao M, Célérier I, Bousquet E, Jeanny JC, Jonet L, Savoldelli M, Offret O, Curan A, Farman N, Jaisser F, et al: Mineralocorticoid receptor is involved in rat and human ocular chorioretinopathy. J Clin Invest 122: 2672-2679, 2012.

37. Ersoz MG, Hocaoglu M, Sayman Muslubas I, Arf S and Karacorlu M: Development of pachychoroid pigment epitheliopathy and transformation to central serous chorioretinopathy after intravitreal dexamethasone implantation. Retin Cases Brief: Sep 26, 2018 (Epub ahead of print). doi: 10.1097/ICB.0000000000000820.

38. Dansingani KK, Perlee LT, Hamon S, Lee M, Shah VP, Spaide RF, Sorenson J, Klancnik JM Jr, Yannuzzi LA, Barbazetto IA, et al: Risk alleles associated with neovascularization in a pachychoroid phenotype. Ophthalmology 123: 2628-2630, 2016.

39. Izumi T, Koizumi H, Maruko I, Takahashi Y, Sonoda S, Sakamoto T and Iida T: Structural analyses of choroid after half-dose verteporfin photodynamic therapy for central serous chorioretinopathy. Br J Ophthalmol 101: 433-437, 2017.

40. Chhablani J, Kozak I, Pichi F, Chenworth M, Berrocal MH, Bedi R, Singh RP, Wu L, Meyerle C, Casella AM, et al; King Khaled Eye Specialist Hospital International Collaborative Retina Study Group: Outcomes of treatment of choroidal neovascularization associated with central serous chorioretinopathy with intravitreal antiangiogenic agents. Retina 35: 2489-2497, 2015.

41. Staurenghi G, Lai TYY, Mitchell P, Wolf S, Wenzel A, Li J, Bhaumik A, Hykin PG; PROMETHEUS Study Group: Efficacy and Safety of Ranibizumab $0.5 \mathrm{mg}$ for the treatment of macular edema resulting from uncommon causes. Twelve-month findings from PROMETHEUS. Ophthalmology 125: 850-862, 2018.

42. Chung YR, Seo EJ, Lew HM and Lee KH: Lack of positive effect of intravitreal bevacizumab in central serous chorioretinopathy: Meta-analysis and review. Eye (Lond) 27: 1339-1346, 2013.

43. Schworm B, Luft N, Keidel LF, Hagenau F, Kern C, Herold T, Kortuem KU, Priglinger SG and Siedlecki J: Response of neovascular central serous chorioretinopathy to an extended upload of anti-VEGF agents. Graefes Arch Clin Exp Ophthalmol 258: 1013-1021, 2020.

44. Sacconi R, Tomasso L, Corbelli E, Carnevali A, Querques L, Casati S, Bandello F and Querques G: Early response to the treatment of choroidal neovascularization complicating central serous chorioretinopathy: A OCT-angiography study. Eye (Lond) 33: 1809-1817, 2019

45. Azuma K, Tan X, Asano S, Shimizu K, Ogawa A, Inoue T, Murata H, Asaoka R and Obata R: The association of choroidal structure and its response to anti-VEGF treatment with the short-time outcome in pachychoroid neovasculopathy. PLoS One 14: e0212055, 2019.

46. Lee JH and Lee WK: One-year results of adjunctive photodynamic therapy for type 1 neovascularization associated with thickened choroid. Retina 36: 889-895, 2016.

47. Koh A, Lee WK, Chen LJ, Chen SJ, Hashad Y, Kim H, Lai TY, Pilz S, Ruamviboonsuk P, Tokaji E, et al: EVEREST study: Efficacy and safety of verteporfin photodynamic therapy in combination with ranibizumab or alone versus ranibizumab monotherapy in patients with symptomatic macular polypoidal choroidal vasculopathy. Retina 32: 1453-1464, 2012.

48. Shin JY, Kwon KY and Byeon SH: Association between choroidal thickness and the response to intravitreal ranibizumab injection in age-related macular degeneration. Acta Ophthalmol 93: 524-532, 2015.

49. Baek J, Lee JH and Lee WK: Clinical relevance of aqueous vascular endothelial growth factor levels in polypoidal choroidal vasculopathy. Retina 37: 943-950, 2017.

50. Saito M, Kano M, Itagaki K, Ise S, Imaizumi K and Sekiryu T: Subfoveal choroidal thickness in polypoidal choroidal vasculopathy after switching to intravitreal aflibercept injection. Jpn J Ophthalmol 60: 35-41, 2016. 
51. Cho HJ, Kim KM, Kim HS, Han JI, Kim CG, Lee TG and Kim JW: Intravitreal aflibercept and ranibizumab injections for polypoidal choroidal vasculopathy. Am J Ophthalmol 165: 1-6, 2016.

52. Koizumi H, Kano M, Yamamoto A, Saito M, Maruko I, Kawasaki R, Sekiryu T, Okada AA and Iida T: Short-term changes in choroidal thickness after aflibercept therapy for neovascular age-related macular degeneration. Am J Ophthalmol 159: 627-633, 2015.

53. Ferrara N: Vascular endothelial growth factor: Basic science and clinical progress. Endocr Rev 25: 581-611, 2004

54. Yong M, Zhou M and Deng G: Photodynamic therapy versus anti-vascular endothelial growth factor agents for polypoidal choroidal vasculopathy: A meta-analysis. BMC Ophthalmol 15: $82,2015$.

55. Stanca HT, Petrović $Z$ and Munteanu M: Transluminal Nd:YAG laser embolysis - a reasonable method to reperfuse occluded branch retinal arteries. Vojnosanit Pregl 71: 1072-1077, 2014
56. Munteanu M, Rosca $\mathrm{C}$ and Stanca H: Sub-inner limiting membrane hemorrhage in a patient with Terson syndrome. Int Ophthalmol 39: 461-464, 2019.

57. Stanca HT, Stanca S, Tabacaru B, Boruga M and Balta F: Bevacizumab in Wet AMD treatment: A tribute to the thirteen years of experience from the beginning of the anti-VEGF era in Romania. Exp Ther Med 18: 4993-5000, 2019.

58. Danielescu C, Stanca HT and Balta F: The management of lamellar macular holes: A review. J Ophthalmol 2020: 3526316 , 2020.

59. Shin JY, Lee JM and Byeon SH: Anti-vascular endothelial growth factor with or without pneumatic displacement for submacular hemorrhage. Am J Ophthalmol 159: 904-14.e1, 2015.

(i) (9) This work is licensed under a Creative Commons Attribution-NonCommercial-NoDerivatives 4.0 International (CC BY-NC-ND 4.0) License. 